\title{
Li in minerals from the Ilímaussaq alkaline intrusion, South Greenland
}

\author{
J. C. BAILEY, H. BOHSE, R. GWOZDZ and J. ROSE-HANSËN
}

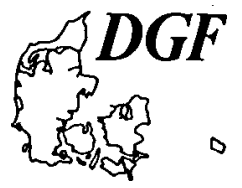

Bailey, J. C., Bohse, H., Gwozdz, R. and Rose-Hansen, J. 1993. Li in minerals from the Ilimaussaq alkaline intrusion, South Greenland. Bulletin of the Geological Society of Denmark, vol. 40, pp. 288-299. Copenhagen 1993-12-30.

https://doi.org/10.37570/bgsd-1993-40-13

\begin{abstract}
$\mathrm{Li}$ was analysed by instrumental neutron activation analysis and Cerenkov counting in 120 mineral samples ( 30 species) from the Ilímaussaq alkaline intrusion, South Greenland. More than 0.23 wt. $\% \mathrm{Li}\left(0.5 \mathrm{wt} \% \mathrm{Li}_{2} \mathrm{O}\right)$ is found in polylithionite, neptunite, riebeckite, $\mathrm{Na}$-cookeite, ephesite, arfvedsonite, gerasimovskite and astrophyllite. Arfvedsonite (200-2500 ppm Li) carries the bulk of $\mathrm{Li}$ in most of the highly alkaline rocks. $\mathrm{Li}-\mathrm{Mg}$ and $\mathrm{Li}-\mathrm{F}$ relations indicate that the distribution of $\mathrm{Li}$ is controlled by the structure of minerals, their absolute contents of $\mathrm{Mg}$ and $\mathrm{F}$ and the fractionation stage within the intrusion. $\mathrm{Li}$ is probably linked with $\mathrm{F}$ in the fluid state and this linkage continues into crystallising phases where $\mathrm{Li}$ occupies sites which also accommodate $\mathrm{Mg}$. $\mathrm{Li} / \mathrm{Mg}$ and $\mathrm{Li} / \mathrm{F}$ ratios of Ilímaussaq rocks and minerals are higher than in equivalent materials from the Lovozero intrusion (Kola, Russia). The Li$\mathrm{Mg}-\mathrm{Fe}^{2+}$ geochemical association at Ilímaussaq $\left(\mathrm{Fe}^{2+}>>\mathrm{Mg}\right)$ and Lovozero $\left(\mathrm{Fe}^{2+}>\mathrm{Mg}\right)$ contrasts with the commercially important $\mathrm{Li}$-rich but $\mathrm{Mg}-\mathrm{Fe}^{2+}$-poor association found in certain granite pegmatites and greisenised granites.
\end{abstract}

Bailey, J.C., Bohse, H., Gwozdz, R. \& Rose-Hansen, J. Division for Petrology, Geological Institute, Øster Voldgade 10, DK-1350 Copenhagen K, Denmark.

Bohse, H. Present address: Mineral Development Int. A/S, Hovedvej 2, DK-2100 Copenhagen, Denmark. Gwozdz, R. Present address: Tracechem, Markmandsgade 2, DK-2300 Copenhagen S, Denmark, June 30th 1992.

\section{Introduction}

Studies of Li distribution among the minerals of igneous rocks have emphasised that $\mathrm{Li}$ is concentrated in $\mathrm{F}$ bearing minerals containing significant levels of $\mathrm{Mg}$, such as micas, amphiboles and tourmaline, and to a lesser extent in Na-rich minerals, such as feldspars (Heier and Adams 1964; Wilson and Long 1983; Henderson et al. 1989). The aim of this publication is to present data on the distribution of $\mathrm{Li}, \mathrm{Mg}$ and $\mathrm{F}$ in minerals of the Ilímaussaq intrusion, South Greenland, and to discuss this distribution in terms of the crystallographic properties of $\mathrm{Li}$ and the petrological history of the intrusion. Comparisons are made with similar materials from the Lovozero intrusion, Russia, and with the commercially important, Li-rich granite pegmatites and greisenised granites.

\section{Geological background}

The Ilímaussaq intrusion (Fig. 1) is the youngest of a number of syenitic bodies in the late Precambrian Gardar province of South Greenland. The intrusion has been described by Ussing (1912), Sørensen (1962, 1969, 1970), Ferguson (1964), Hamilton (1964), Bohse et al. (1971), Engell (1973), Larsen (1976), Bailey et al. (1981a) and Larsen and Sørensen (1987). It consists of an outer envelope of augite syenite (phase 1) and two thin sheets of peralkaline granite under the roof (phase 2) which were followed by a layered series of peralkaline nepheline syenites (phase 3). The flotation cumulates of the layered series are rich in sodalite (sodalite foyaite, naujaite) whereas the bottom cumulates (kakortokites) contain abundant arfvedsonite, eudialyte and microcline. The intervening, residual lujavrites were emplaced in the sequence: aegirine lujavrite I, aegirine lujavrite II, lujavrite transition zone, arfvedsonite lujavrite and then medium- to coarse-grained (M-C) lujavrite. Locally, there are lujavrite varieties 


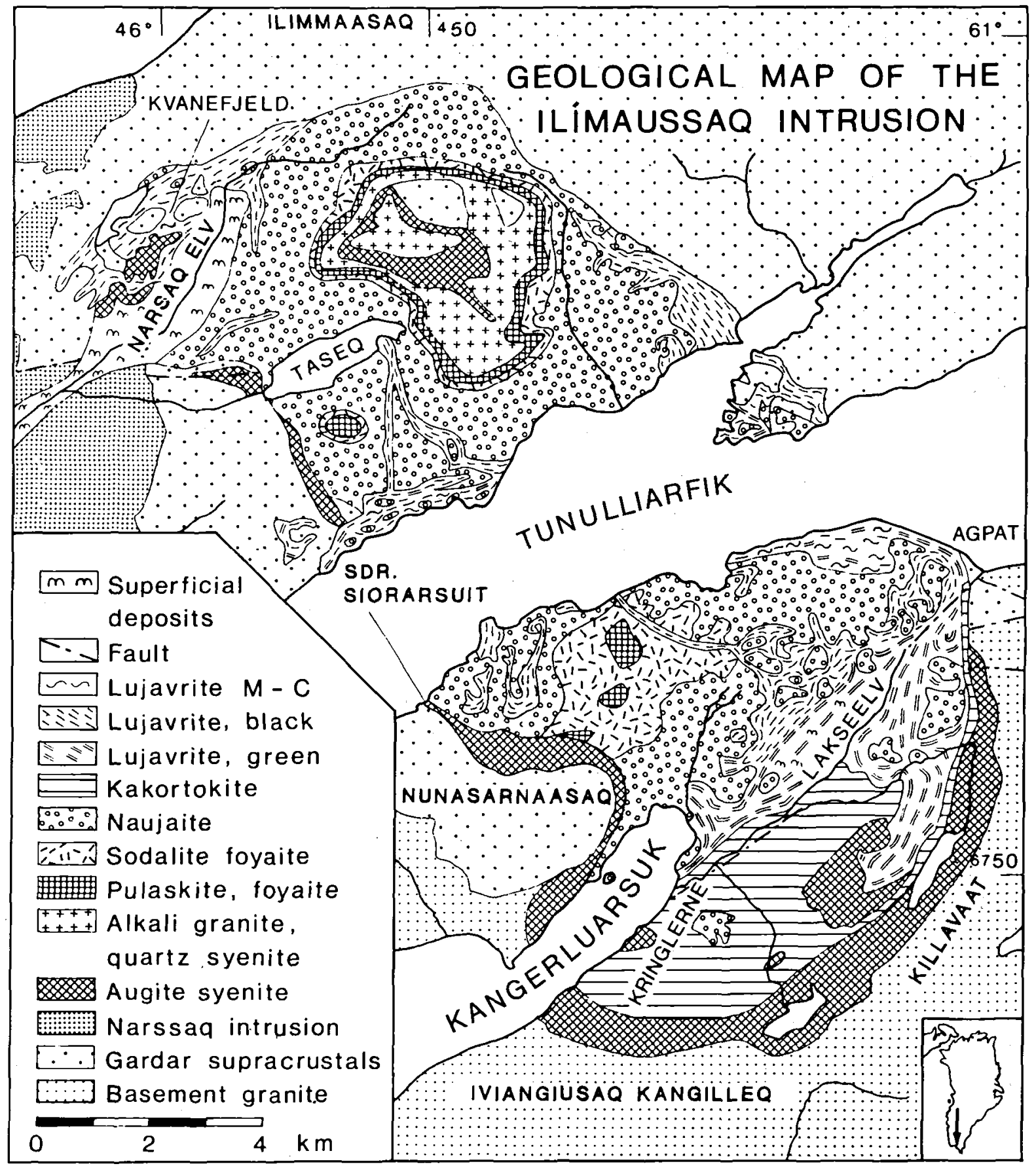

Fig. 1. Geological summary map of the Ilímaussaq intrusion based on Ferguson (1964).

which are rich in naujakasite and villiaumite and, in the roof zone, the lujavrites have notable contents of $U$ and Th (Sørensen et al. 1974).

The minerals investigated were mainly taken from the peralkaline nepheline syenites and their pegmatitic and hydrothermal derivatives.

Bulletin of the Geological Society of Denmark

\section{Existing data}

The Ilimaussaq intrusion constitutes a major $\mathbf{L i}$ anomaly in the world of igneous rocks. Li contents in chilled marginal augite syenite are only about $23 \mathrm{ppm}$ 
Table 1. Previously published contents of $\mathrm{Li}, \mathrm{F}$ and $\mathrm{Mg}$ in Ilímaussaq minerals.

\begin{tabular}{|c|c|c|c|c|c|c|}
\hline \multirow[t]{2}{*}{ Mineral } & \multicolumn{2}{|c|}{$\mathrm{Li}(\mathrm{ppm})$} & \multicolumn{2}{|c|}{$F(\%)$} & \multicolumn{2}{|c|}{$\operatorname{Mg}(\%)$} \\
\hline & range & median & range & median & range & median \\
\hline Aegirine & $20-160$ & 50 & - & - & $0.00-0.87$ & 0.11 \\
\hline Albite & $1-18$ & 9 & - & - & - & - \\
\hline Analcime & $6-7$ & 6.5 & - & - & - & - \\
\hline Arfvedsonite & $100-1015$ & 940 & $0.29-1.23$ & 0.88 & $0.02-0.72$ & 0.19 \\
\hline Biotite-lepidomelane & n00 & no0 & - & - & - & - \\
\hline Katophorite & 300 & 330 & n.d. -0.80 & 0.40 & $0.15-0.98$ & 0.57 \\
\hline Chkalovite & $0.5-10$ & 5 & - & - & n.d. -0.10 & 0.05 \\
\hline Na-Cookeite & 2300 & 2300 & - & - & - & - \\
\hline Eudialyte & $10-330$ & 60 & 0.28 & 0.28 & $0.05-0.16$ & 0.09 \\
\hline Feldspar & 25 & 25 & - & - & - & - \\
\hline Mafics & 160 & 160 & - & - & - & - \\
\hline Microline & $5-17$ & 11 & - & - & tr. & tr. \\
\hline Microline + nepheline & 11 & 11 & - & - & - & - \\
\hline Neptunite & 6600 & 6600 & - & - & 0.18 & 0.18 \\
\hline Polylithionite & $2.72-4.34 \%$ & $3.15 \%$ & $6.4-8.59$ & 7.61 & n.d. -0.04 & 0.02 \\
\hline Potash feldspar & 2 & 2 & - & - & - & - \\
\hline Riebeckite & $1500-3900$ & 2700 & 0.40 & 0.40 & 0.16 & 0.16 \\
\hline Sodalite & $15-90$ & 32 & - & - & - & - \\
\hline Steenstrupine & $100-300$ & 200 & $0.27-1.32$ & 1.24 & $0.03-0.19$ & 0.05 \\
\hline Ussingite & 30 & 30 & 0.09 & 0.09 & 0.34 & 0.34 \\
\hline
\end{tabular}

-: data unavailable, tr.: trace, n.d.: not detected

Results compiled from: Bøggild (1953), Sørensen (1962), Hamilton (1964), Gerasimovsky (1969), Semenov 1969), Povarennykh et al. (1970), Engell (1973), Sørensen et al. (1974), Larsen (1976), Makovicky and Karup-Møller (1981).

but rise to $90-300 \mathrm{ppm}$ in subsequent agpaitic rocks (sodalite foyaite, naujaite, kakortokite, aegirine lujavrite) and to $600-1000 \mathrm{ppm}$ in the residual arfvedsonite lujavrites (Gerasimovsky 1969; Bailey et al. 1981b). Concentrations of several thousand ppm probably occur in scattered pegmatites containing Li-mica.

The literature contains $\mathrm{Li}$ results on 45 mineral samples (20 species) from Ilímaussaq (Table 1). Data for $\mathrm{Li}$, $\mathrm{Mg}$ and $\mathrm{F}$ have only occasionally been determined on single mineral specimens. The variety of analytical techniques employed explains some of the observed range of values. The presence of impurities in mineral separates has received no (published) attention, though the widespread occurrence of $\mathrm{Li}$-rich phases indicates that this is a potential cause of serious errors.

Eight Ilímaussaq minerals contain $0.23 \mathrm{wt}$ \% or more of $\mathrm{Li}$ (equivalent to $0.50 \mathrm{wt}$ \% or more of $\left.\mathrm{Li}_{2} \mathrm{O}\right)(\mathrm{B} \emptyset \mathrm{g}$ gild 1953; Sørensen 1962; Semenov 1969; this paper).

Polylithionite, or according to more recent studies lepidolite, $\mathrm{KLi}_{2} \mathrm{Al}\left|(\mathrm{F}, \mathrm{OH})_{2}\right| \mathrm{Si}_{4} \mathrm{O}_{10} \mid$, is a widespread accessory mineral in naujaite and lujavrite and their derivatives. It also occurs in foyaite, kakortokite and in quartz-feldspar veins cutting alkali granite. High temperature polylithionite is lamellar and occurs in rocks and pegmatites. Flaky varieties are found in the central zones of pegmatites and in albitization zones. Earthy polylithionite occurs in low temperature hydrothermal zeolite vugs and contains $2.72 \% \mathrm{Li}$ in contrast to $3.0-4.2 \%$ in the earlier varieties (Semenov 1969).

Neptunite, $\mathrm{KNa}_{2} \mathrm{Li}(\mathrm{Fe}, \mathrm{Mn})_{2} \mathrm{Ti}_{2}\left|\mathrm{OSi}_{4} \mathrm{O}_{11}\right|_{2}$, is most characteristic for the naujaite and $\mathrm{M}-\mathrm{C}$ lujavrite hy- drothermal veins but also occurs in quartz veins in alkali granite and in cross-cutting kakortokite pegmatites. It is typically associated with altered arfvedsonite and eudialyte. $\mathrm{Li}(0.66 \%)$ does not substitute for Fe or alkalis but is an essential component of the neptunite formula (Semenov 1969).

Riebeckite, $\mathrm{Na}_{2} \mathrm{Fe}_{3}{ }^{+2} \mathrm{Fe}_{2}{ }^{+3} \mathrm{Si}_{8} \mathrm{O}_{22}(\mathrm{OH}, \mathrm{F})_{2}$, is common in late, usually hydrothermal derivatives of naujaite and alkali granite. $\mathrm{Li}$ contents range from $0.15-0.39 \% . \mathrm{Li}^{+}$ may substitute for $\mathrm{Fe}^{2+}$ (Semenov 1969).

Na-cookeite, $\left.\quad \mathrm{Al}_{2}\left|(\mathrm{OH})_{2}\right| \mathrm{AlSi}_{3} \mathrm{O}_{10}\right|^{1}-\mathrm{LiAl}_{2}(\mathrm{OH})_{6}{ }^{1+}$, occurs in the central hydrothermal zone of an $\mathrm{M}-\mathrm{C}$ lujavrite pegmatite in the Narssaq river (Semenov 1969). It is $\mathrm{Li}$-poor $(0.23 \%)$ and Na-rich compared with previously described cookeites, and is probably a special polymorphic modification of cookeite.

Ephesite, $\mathrm{NaLiAl}_{2}\left|(\mathrm{OH})_{2}\right| \mathrm{Al}_{2} \mathrm{Si}_{2} \mathrm{O}_{10}$, with $1.21 \% \mathrm{Li}$ also occurs in the hydrothermal zone of an M-C lujavrite pegmatite at Narssaq river (Semenov 1969; Drits and Semenov 1975). Ephesite is a member of the margarite group and $\mathrm{Li}$ fills the vacant octahedral position as $\mathrm{Ca}^{2+}$ is replaced by $\mathrm{Na}^{+}$(Schaller et al. 1967).

These five minerals are mainly developed at the hydrothermal stage. Arfvedsonite, $\mathrm{Na}_{3} \mathrm{Fe}_{4}{ }^{2+} \mathrm{Fe}^{3+} \mathrm{Si}_{8} \mathrm{O}_{22}$ $(\mathrm{OH})_{2}$, however, is an abundant rock-forming mineral in the peralkaline rocks and their derivatives. Only in aegirine lujavrite I, the earliest of the lujavrite varieties, do $\mathrm{Li}$ values in arfvedsonite rise above $0.23 \%$. The poikilitic arfvedsonite, up to $8 \mathrm{vol} . \%$, is considered to have crystallised from a Li-rich interstitial coexisting magma (Bailey and Gwozdz, submitted). 
Table 2. Concentration of $\mathrm{Li}, \mathrm{F}$ and $\mathrm{Mg}$ in Ilimaussaq minerals. Li and $\mathrm{F}$ by instrumental neutron activation analysis and Cerenkov counting; $\mathrm{Mg}$ by X-ray fluorescence analysis.

\begin{tabular}{|c|c|c|c|c|c|c|c|}
\hline Mineral & Sample number & Rock type & Local. $^{+)}$ & Collec. $^{++)}$ & $\begin{array}{c}\mathbf{L i} \\
\mathbf{p p m}\end{array}$ & $\begin{array}{l}\mathbf{F} \\
\%\end{array}$ & $\underset{\%}{\mathrm{Mg}}$ \\
\hline $\begin{array}{l}\text { Acmite } \\
\text { Acmite }\end{array}$ & $\begin{array}{l}21070 \mathrm{~A} \\
21070\end{array}$ & $\begin{array}{l}\text { Aegirine lujavrite } \\
\text { Aegirine lujavrite }\end{array}$ & $\begin{array}{l}\mathbf{K} \\
\mathbf{K}\end{array}$ & $\begin{array}{l}\mathrm{OL} \\
\mathrm{OL}\end{array}$ & $\begin{array}{l}26 \\
47\end{array}$ & $\begin{array}{l}\text { n.d. } \\
\text { n.d. }\end{array}$ & $\begin{array}{l}\text { n.d. } \\
0.04\end{array}$ \\
\hline $\begin{array}{l}\text { Aegirine } \\
\text { Aegirine } \\
\text { Aegirine } \\
\text { Aegirine } \\
\text { Aegirine } \\
\text { Aegirine } \\
\text { Aegirine } \\
\text { Aegirine } \\
\text { Aegirine } \\
\text { Aegirine } \\
\text { Aegirine } \\
\text { Aegirine } \\
\text { Aegirine } \\
\text { Aegirine } \\
\text { Aegirine }\end{array}$ & $\begin{array}{l}108107-191^{*} \\
108107-166 \\
108107-146 \\
108107-127 \\
108107-106 \\
108107-096 \\
108107-069 \\
108107-046 \\
108107-022 \\
108107-004 \\
149541 \\
21070 \\
21178 \\
18453 \\
65930\end{array}$ & $\begin{array}{l}\text { Aegirine lujavrite I } \\
\text { Aegirine lujavrite I } \\
\text { Aegirine lujavrite I } \\
\text { Aegirine lujavrite I } \\
\text { Aegirine lujavrite I } \\
\text { Aegirine lujavrite I } \\
\text { Aegirine lujavrite I } \\
\text { Aegirine lujavrite I } \\
\text { Aegirine lujavrite I } \\
\text { Aegirine lujavrite I } \\
\text { Lujavrite pegmatite } \\
\text { Aegirine lujavrite } \\
\text { Aegirine lujavrite } \\
\text { Aegirine lujavrite } \\
\text { Hydrothermal vein }\end{array}$ & $\begin{array}{c}\mathbf{K} \\
\mathbf{K} \\
\mathbf{K} \\
\mathbf{K} \\
\mathbf{K} \\
\mathbf{K} \\
\mathbf{K} \\
\mathbf{K} \\
\mathbf{K} \\
\mathbf{K} \\
\mathrm{Kv} \\
\mathrm{Tu} \\
\mathbf{K} \\
\mathrm{Kv} \\
\mathrm{T}\end{array}$ & $\begin{array}{l}\text { OL } \\
\text { OL } \\
\text { JE }\end{array}$ & $\begin{array}{l}10 \\
26 \\
21 \\
30 \\
32 \\
16 \\
37 \\
13 \\
17 \\
27 \\
25 \\
23 \\
33 \\
16 \\
24\end{array}$ & $\begin{array}{l}\text { n.d. } \\
\text { n.d. } \\
\text { n.d. } \\
\text { n.d. } \\
\text { n.d. } \\
\text { n.d. } \\
\text { n.d. } \\
\text { n.d. } \\
\text { n.d. } \\
\text { n.d. } \\
\text { n.d. } \\
\text { n.d. } \\
\text { n.d. } \\
\text { n.d. } \\
\text { n.d. }\end{array}$ & $\begin{array}{l}0.02 \\
0.03 \\
0.03 \\
0.02 \\
0.03 \\
0.03 \\
0.02 \\
0.03 \\
0.04 \\
0.03 \\
0.04 \\
0.02 \\
0.02 \\
0.05 \\
-\end{array}$ \\
\hline $\begin{array}{l}\text { Analcime } \\
\text { Analcime }\end{array}$ & $\begin{array}{l}71 \\
65914\end{array}$ & $\begin{array}{l}\text { Steenstrupine-analcime } \\
\text { arfvedsonite pegmatite } \\
\text { Analcime vein }\end{array}$ & $\begin{array}{l}\mathrm{K} \\
\mathrm{T}\end{array}$ & $\begin{array}{l}\mathrm{HS} \\
\mathrm{JE}\end{array}$ & $\begin{array}{c}12 \\
3.8\end{array}$ & $\begin{array}{l}0.06 \\
0.13\end{array}$ & $\begin{array}{l}0.01 \\
0.01\end{array}$ \\
\hline $\begin{array}{l}\text { Analcime + natrolite } \\
\text { Analcime + natrolite } \\
\text { Analcime + natrolite } \\
\text { Analcime + natrolite } \\
\text { Analcime + natrolite } \\
\text { Analcime + natrolite } \\
\text { Analcime + natrolite } \\
\text { Analcime + natrolite } \\
\text { Analcime + natrolite }\end{array}$ & $\begin{array}{l}108107-191 \\
108107-166 \\
108107-146 \\
108107-127 \\
108107-106 \\
108107-096 \\
108107-069 \\
108107-046 \\
108107-022\end{array}$ & $\begin{array}{l}\text { Aegirine lujavrite I } \\
\text { Aegirine lujavrite I } \\
\text { Aegirine lujavrite I } \\
\text { Aegirine lujavrite I } \\
\text { Aegirine lujavrite I } \\
\text { Aegirine lujavrite I } \\
\text { Aegirine lujavrite I } \\
\text { Aegirine lujavrite I } \\
\text { Aegirine lujavrite I }\end{array}$ & $\begin{array}{l}\mathrm{K} \\
\mathrm{K} \\
\mathrm{K} \\
\mathrm{K} \\
\mathbf{K} \\
\mathrm{K} \\
\mathrm{K} \\
\mathrm{K} \\
\mathrm{K}\end{array}$ & & $\begin{array}{l}32 \\
24 \\
30 \\
27 \\
38 \\
21 \\
23 \\
15 \\
21\end{array}$ & $\begin{array}{l}\text { n.d. } \\
\text { n.d. } \\
0.11 \\
\text { n.d. } \\
\text { n.d. } \\
\text { n.d. } \\
\text { n.d. } \\
\text { n.d. } \\
\text { n.d. }\end{array}$ & $\begin{array}{l}\text { n.d. } \\
0.01 \\
0.01 \\
0.01 \\
0.01 \\
0.02 \\
0.02 \\
0.03 \\
0.02\end{array}$ \\
\hline $\begin{array}{l}\text { Arfvedsonite } \\
\text { Arfvedsonite } \\
\text { Arfvedsonite } \\
\text { Arfvedsonite } \\
\text { Arfvedsonite } \\
\text { Arfvedsonite } \\
\text { Arfvedsonite } \\
\text { Arfvedsonite } \\
\text { Arfvedsonite }\end{array}$ & $\begin{array}{l}154351 \\
149594 \\
154717 \mathrm{~B} \\
154309 \\
57041 \\
154331 \\
108133 \\
108131 \\
108107-33.7\end{array}$ & $\begin{array}{l}\text { Sodalite foyaite } \\
\text { Naujaite pegmatite } \\
\text { Naujaite pegmatite } \\
\text { Naujaite } \\
\text { Naujaite } \\
\text { Naujaite } \\
\text { Black lujavrite } \\
\text { Black lujavrite } \\
\text { Naujaite xenolith in } \\
\text { aegirine lujavrite I }\end{array}$ & $\begin{array}{l}\mathbf{K} \\
\mathbf{K} \\
\mathbf{K} \\
\mathbf{K} \\
\mathbf{K} \\
\mathbf{K} \\
\mathbf{K} \\
\mathbf{K} \\
\\
\mathbf{K}\end{array}$ & HM & $\begin{array}{l}489 \\
281 \\
243 \\
255 \\
462 \\
420 \\
289 \\
370 \\
\\
233\end{array}$ & $\begin{array}{l}0.57 \\
0.59 \\
- \\
0.24 \\
0.55 \\
0.68 \\
0.36 \\
0.17 \\
\\
0.15\end{array}$ & $\begin{array}{c}0.05 \\
0.21 \\
- \\
\text { n.d. } \\
0.05 \\
0.08 \\
0.04 \\
0.06 \\
\\
0.51\end{array}$ \\
\hline $\begin{array}{l}\text { Arfvedsonite } \\
\text { Arfvedsonite } \\
\text { Arfvedsonite } \\
\text { Arfvedsonite } \\
\text { Arfvedsonite } \\
\text { Arfvedsonite } \\
\text { Arfvedsonite } \\
\text { Arfvedsonite } \\
\text { Arfvedsonite } \\
\text { Arfvedsonite } \\
\text { Arfvedsonite } \\
\text { Arfvedsonite } \\
\text { Arfvedsonite } \\
\text { Arfvedsonite } \\
\text { Arfvedsonite }\end{array}$ & $\begin{array}{l}154343 \\
109201 \\
109240 \\
154321 \\
108107-191 \\
108107-166 \\
108107-146 \\
108107-127 \\
108107-106 \\
108107-096 \\
108107-069 \\
108107-046 \\
108107-022 \\
108107-004 \\
117479\end{array}$ & $\begin{array}{l}\text { Black kakortokite } \\
\text { Black kakortokite } \\
\text { Red kakortokite } \\
\text { Black kakortokite } \\
\text { Aegirine lujavrite I } \\
\text { Aegirine lujavrite I } \\
\text { Aegirine lujavrite I } \\
\text { Aegirine lujavrite I } \\
\text { Aegirine lujavrite I } \\
\text { Aegirine lujavrite I } \\
\text { Aegirine lujavrite I } \\
\text { Aegirine lujavrite I } \\
\text { Aegirine lujavrite I } \\
\text { Aegirine lujavrite I } \\
\text { Lujavrite-naujaite } \\
\text { contact negmatite }\end{array}$ & $\begin{array}{l}\mathrm{K} \\
\mathbf{K} \\
\mathbf{K} \\
\mathrm{K} \\
\mathrm{K} \\
\mathrm{K} \\
\mathbf{K} \\
\mathrm{K} \\
\mathrm{K} \\
\mathrm{K} \\
\mathbf{K} \\
\mathbf{K} \\
\mathbf{K} \\
\mathbf{K}\end{array}$ & & $\begin{array}{r}513 \\
378 \\
410 \\
725 \\
2020 \\
2200 \\
1990 \\
2520 \\
2200 \\
2090 \\
2250 \\
1980 \\
2350 \\
1970\end{array}$ & $\begin{array}{l}0.47 \\
0.45 \\
0.33 \\
0.20 \\
0.16 \\
0.27 \\
0.28 \\
0.23 \\
0.22 \\
0.18 \\
0.22 \\
0.16 \\
0.28 \\
0.24\end{array}$ & $\begin{array}{c}- \\
- \\
-\overline{0} \\
0.12 \\
0.04 \\
0.05 \\
0.04 \\
0.05 \\
- \\
0.04 \\
0.04 \\
0.06 \\
0.09 \\
0.04\end{array}$ \\
\hline $\begin{array}{l}\text { Arfvedsonite } \\
\text { Arfvedsonite }\end{array}$ & $\begin{array}{l}65132 \\
.163055\end{array}$ & $\begin{array}{l}\text { Sheared country rock } \\
\text { Central vein in } \\
\text { pegmatite }\end{array}$ & $\begin{array}{c}\mathrm{K} \\
\mathrm{Kv} \\
\mathrm{K}\end{array}$ & & $\begin{array}{l}1240 \\
2010 \\
1680\end{array}$ & $\begin{array}{l}0.28 \\
0.42 \\
0.33\end{array}$ & $\begin{array}{l}0.09 \\
1.7 \\
0.06\end{array}$ \\
\hline Arfvedsonite & 71 & $\begin{array}{l}\text { Steenstrupine-analcime } \\
\text { arfvedsonite pegmatite }\end{array}$ & $\mathrm{K}$ & HS & 1770 & 0.08 & 0.05 \\
\hline
\end{tabular}


Table 2. - Cont.

\begin{tabular}{|c|c|c|c|c|c|c|c|}
\hline Mineral & Sample number & Rock type & Local. ${ }^{+)}$ & Collec $^{++)}$ & $\underset{\mathrm{ppm}}{\mathrm{Li}}$ & $\begin{array}{l}\mathbf{F} \\
\%\end{array}$ & $\underset{\%}{\mathrm{Mg}}$ \\
\hline $\begin{array}{l}\text { Arfvedsonite } \\
\text { Arfvedsonite } \\
\text { Arfvedsonite }\end{array}$ & $\begin{array}{l}154370 \\
119437-092.28 \\
154399\end{array}$ & $\begin{array}{l}\text { Aegirine lujavrite } \\
\text { M-C lujavrite } \\
\text { M-C lujavrite }\end{array}$ & $\begin{array}{l}\text { Tu } \\
\text { Kv } \\
\text { Kv }\end{array}$ & & $\begin{array}{r}459 \\
1570 \\
1490\end{array}$ & $\begin{array}{l}0.37 \\
0.09 \\
0.20\end{array}$ & $\begin{array}{c}0.13 \\
0.02 \\
-\end{array}$ \\
\hline $\begin{array}{l}\text { Astrophyllite } \\
\text { (Nb-rich) }\end{array}$ & $119411-7265$ & Hydrothermal vein & $\mathrm{Kv}$ & & 3000 & 0.71 & 2.9 \\
\hline Astrophyllite & $\mathrm{L} \emptyset \mathrm{K}$ AST & Hydrothermal vein & $\mathbf{K}$ & LL & 223 & 1.07 & 0.12 \\
\hline Beta-natrolite & - & Hydrothermal vein & $\mathrm{T}$ & & 1.7 & n.d. & 0.01 \\
\hline Chkalovite & 155233 & Ussingite vein & $\mathrm{T}$ & & 8.2 & n.d. & n.d. \\
\hline $\begin{array}{l}\text { Epistolite } \\
\text { Epistolite } \\
\text { Epistolite } \\
\text { Epistolite }\end{array}$ & $\begin{array}{l}108130 \\
155231 \\
77512\end{array}$ & $\begin{array}{l}\text { Ussingite vein } \\
\text { Ussingite vein } \\
\text { Ussingite vein } \\
\text { Ussingite vein }\end{array}$ & $\begin{array}{l}\mathrm{T} \\
\mathrm{T} \\
\mathrm{T} \\
\mathrm{Kv}\end{array}$ & & $\begin{array}{r}36 \\
38 \\
49 \\
421\end{array}$ & $\begin{array}{l}0.77 \\
0.97 \\
0.62 \\
0.13\end{array}$ & $\begin{array}{l}0.08 \\
0.09 \\
0.17 \\
0.18\end{array}$ \\
\hline $\begin{array}{l}\text { Eudialyte } \\
\text { Eudialyte } \\
\text { Eudialyte } \\
\text { Eudialyte } \\
\text { Eudialyte } \\
\text { Eudialyte } \\
\text { Eudialyte } \\
\text { Eudialyte } \\
\text { Eudialyte } \\
\text { Eudialyte } \\
\text { Eudialyte } \\
\text { Eudialyte } \\
\text { Eudialyte } \\
\text { Eudialyte } \\
\text { Eudialyte } \\
\text { Eudialyte } \\
\text { Eudialyte } \\
\text { Eudialyte } \\
\text { Eudialyte }\end{array}$ & $\begin{array}{l}154352 \\
154303 \\
60755 \\
57041 \\
154331 \\
108107-033.7 \\
\\
109269 \\
109202 \\
117815 \\
108136 \\
143610 \\
108107-191 \\
108107-166 \\
108107-146 \\
108107-096 \\
108107-046 \\
108107-022 \\
108107-004 \\
57033\end{array}$ & $\begin{array}{l}\text { Sodalite foyaite } \\
\text { Sodalite foyaite } \\
\text { Kakortokite } \\
\text { Naujaite } \\
\text { Naujaite } \\
\text { Naujaite xenolith in } \\
\text { aegirine lujavrite I } \\
\text { Red kakortokite } \\
\text { Red kakortokite } \\
\text { Red kakortokite } \\
\text { Red kakortokite } \\
\text { Feldspar-eudialyte vein } \\
\text { Aegirine lujavrite I } \\
\text { Aegirine lujavrite I } \\
\text { Aegirine lujavrite I } \\
\text { Aegirine lujavrite I } \\
\text { Aegirine lujavrite I } \\
\text { Aegirine lujavrite I } \\
\text { Aegirine lujavrite I } \\
\text { Aegirine lujavrite I }\end{array}$ & $\begin{array}{l}\mathrm{Tu} \\
\mathrm{Tu} \\
\mathrm{K} \\
\mathrm{K} \\
\mathrm{K} \\
\mathrm{K} \\
\mathrm{K} \\
\mathrm{K} \\
\mathrm{K} \\
\mathrm{K} \\
\mathrm{K} \\
\mathrm{K} \\
\mathrm{K} \\
\mathrm{K} \\
\mathrm{K} \\
\mathrm{K} \\
\mathrm{K} \\
\mathrm{K} \\
\mathrm{K}\end{array}$ & HM & $\begin{array}{c}268 \\
174 \\
61 \\
13 \\
12 \\
17 \\
8.2 \\
9.7 \\
7.1 \\
18 \\
8.4 \\
8.6 \\
8.4 \\
8.5 \\
7.5 \\
13 \\
15 \\
32 \\
10\end{array}$ & $\begin{array}{l}0.10 \\
\text { n.d. } \\
\text { n.d. } \\
0.06 \\
\text { n.d. } \\
\text { n.d. } \\
\text { n.d. } \\
\text { n.d. } \\
\text { n.d. } \\
0.18 . \\
\text { n.d. } \\
\text { n.d. } \\
\text { n.d. } \\
0.05 \\
\text { n.d. } \\
0.12 \\
0.14 \\
0.09 \\
\text { n.d. }\end{array}$ & $\begin{array}{c}0.02 \\
\text { n.d. } \\
\text { n.d. } \\
\text { n.d. } \\
0.01 \\
- \\
0.03 \\
0.02 \\
0.02 \\
0.02 \\
0.04 \\
0.04 \\
0.02 \\
0.01\end{array}$ \\
\hline Gerasimovskite & 77480 & Hydrothermal vein & $\mathrm{T}$ & & 12400 & 2.34 & 0.01 \\
\hline Igdloite & 185101 & Hydrothermal vein & $\mathrm{Kv}$ & & 20 & 0.14 & 0.05 \\
\hline Kaersutite & 77102 & Lamprophyre & $\mathrm{Kv}$ & & 106 & 0.09 & 3.0 \\
\hline $\begin{array}{l}\text { Microcline + nepheline } \\
\text { Microcline + nepheline } \\
\text { Microcline + nepheline } \\
\text { Microcline + nepheline } \\
\text { Microcline + nepheline }\end{array}$ & $\begin{array}{l}108107-191 \\
108107-146 \\
108107-096 \\
108107-046 \\
108107-022\end{array}$ & $\begin{array}{l}\text { Aegirine lujavrite I } \\
\text { Aegirine lujavrite I } \\
\text { Aegirine lujavrite I } \\
\text { Aegirine lujavrite I } \\
\text { Aegirine lujavrite I }\end{array}$ & $\begin{array}{l}\mathrm{K} \\
\mathrm{K} \\
\mathrm{K} \\
\mathrm{K} \\
\mathrm{K}\end{array}$ & & $\begin{array}{l}5.9 \\
3.0 \\
8.2 \\
4.9 \\
2.0\end{array}$ & $\begin{array}{l}\text { n.d. } \\
\text { n.d. } \\
\text { n.d. } \\
\text { n.d. } \\
\text { n.d. }\end{array}$ & $\begin{array}{l}\text { n.d. } \\
0.01 \\
0.01 \\
0.01 \\
\text { n.d. }\end{array}$ \\
\hline Murmanite & 65127 & Sheared country rock & $\mathrm{Kv}$ & & 346 & 0.13 & - \\
\hline Naujakasite & $149421 \mathrm{C}$ & Naujakasite lujavrite & $\mathrm{Kv}$ & & 5 & - & 0.07 \\
\hline Nenadkevichite & $\mathrm{L} \emptyset \mathrm{K} 27 \mathrm{~A}$ & Hydrothermal vein & $\mathrm{T}$ & $\mathrm{LL}$ & 306 & 0.62 & 0.04 \\
\hline $\begin{array}{l}\text { Neptunite } \\
\text { Neptunite }\end{array}$ & $\begin{array}{l}65450 \\
65450\end{array}$ & $\begin{array}{l}\text { Hydrothermal vein } \\
\text { Hydrothermal vein }\end{array}$ & $\begin{array}{l}\mathbf{T} \\
\mathrm{T}\end{array}$ & & $\begin{array}{l}5440 \\
6700\end{array}$ & $\begin{array}{l}0.09 \\
0.14\end{array}$ & $\begin{array}{c}\text { n.d. } \\
-\end{array}$ \\
\hline $\begin{array}{l}\text { Niobophyllite } \\
\text { Niobophyllite }\end{array}$ & $\begin{array}{l}65112 \mathrm{P} \\
65112 \mathrm{P}\end{array}$ & $\begin{array}{l}\text { Sheared country rock } \\
\text { Hydrothermal vein }\end{array}$ & $\begin{array}{l}\mathrm{Kv} \\
\mathrm{Kv}\end{array}$ & & $\begin{array}{l}157 \\
218\end{array}$ & $\begin{array}{l}1.81 \\
0.58\end{array}$ & n.d. \\
\hline $\begin{array}{l}\text { Polylithionite } \\
\text { Polylithionite } \\
\text { Polylithionite } \\
\text { Polylithionite } \\
\text { Polylithionite } \\
\text { Polylithionite } \\
\text { Polylithionite } \\
\text { Polylithionite }\end{array}$ & $\begin{array}{l}64684 \\
154717 \mathrm{~A} \\
\text { QEQ } \\
150318 \\
18617 \\
64684 \mathrm{~A} \\
20770 \\
\text { LøK } 13 \mathrm{R}\end{array}$ & $\begin{array}{l}\text { Naujaite pegmatite } \\
\text { Naujaite pegmatite } \\
\text { Naujaite pegmatite } \\
\text { Naujaite pegmatite } \\
\text { Naujaite pegmatite } \\
\text { Naujaite pegmatite } \\
\text { Naujaite pegmatite } \\
\text { Kakortokite pegmatite }\end{array}$ & $\begin{array}{l}\mathrm{Kv} \\
\mathrm{K} \\
\mathrm{K} \\
\mathrm{K} \\
\mathrm{K} \\
\mathrm{Kv} \\
\mathrm{K} \\
\mathrm{K}\end{array}$ & $\therefore \quad \mathrm{LL}$ & $\begin{array}{l}35400 \\
33000 \\
32400 \\
32000 \\
31300 \\
31000 \\
29700 \\
34500\end{array}$ & $\begin{array}{c}4.75 \\
- \\
- \\
4.59 \\
3.89 \\
4.97 \\
4.48 \\
-\end{array}$ & $\begin{array}{l}\text { n.d. } \\
0.02 \\
0.04 \\
\text { n.d. } \\
0.02 \\
0.06 \\
0.03 \\
0.06\end{array}$ \\
\hline
\end{tabular}


Table 2. - Cont.

\begin{tabular}{|c|c|c|c|c|c|c|c|}
\hline Mineral & Sample number & Rock type & Local. $^{+)}$ & Collec. ${ }^{++)}$ & $\underset{\mathrm{ppm}}{\mathbf{L i}}$ & $\underset{\%}{\mathrm{~F}}$ & $\underset{\%}{\mathrm{Mg}}$ \\
\hline $\begin{array}{l}\text { Pyrochlore } \\
\text { Pyrochlore } \\
\text { Pyrochlore } \\
\text { Pyrochlore } \\
\text { Pyrochlore }\end{array}$ & $\begin{array}{l}65468 \\
65450(2) \\
65450(5) \\
65450 \\
65451\end{array}$ & $\begin{array}{l}\text { Hydrothermal vein } \\
\text { Hydrothermal vein } \\
\text { Hydrothermal vein } \\
\text { Hydrothermal vein } \\
\text { Hydrothermal vein }\end{array}$ & $\begin{array}{l}\mathrm{Kv} \\
\mathrm{Kv} \\
\mathrm{Kv} \\
\mathrm{Kv} \\
\mathrm{Kv}\end{array}$ & & $\begin{array}{r}52 \\
80 \\
101 \\
226 \\
257\end{array}$ & $\begin{array}{l}1.41 \\
1.57 \\
1.81 \\
1.00 \\
1.66\end{array}$ & $\begin{array}{l}0.02 \\
0.02 \\
0.01 \\
\text { n.d. } \\
0.02\end{array}$ \\
\hline Quartz & 152140 & Alkali granite & $\mathrm{Kv}$ & & 1.2 & n.d. & 0.01 \\
\hline $\begin{array}{l}\text { Sodalite } \\
\text { Sodalite } \\
\text { Sodalite }\end{array}$ & $\begin{array}{l}154331 \\
108107-033.7 \\
108118\end{array}$ & $\begin{array}{l}\text { Naujaite } \\
\text { Naujaite xenolith in } \\
\text { aegirine lujavrite I } \\
\text { Hydrothermal vein }\end{array}$ & $\begin{array}{c}\mathrm{K} \\
\mathrm{Kv}\end{array}$ & & $\begin{array}{r}131 \\
33 \\
24\end{array}$ & $\begin{array}{l}0.80 \\
\text { n.d. } \\
\text { n.d. }\end{array}$ & $\begin{array}{l}- \\
0.01 \\
0.04\end{array}$ \\
\hline Sorensenite & 155232 & Hydrothermal vein & $\mathbf{K v}$ & & 11 & n.d. & 0.05 \\
\hline $\begin{array}{l}\text { Steenstrupine } \\
\text { Steenstrupine } \\
\text { Steenstrupine }\end{array}$ & $\begin{array}{l}71 \\
119408-33.52 \\
18467\end{array}$ & $\begin{array}{l}\text { Steenstrupine-analcime } \\
\text { arfvedsonite pegmatite } \\
\text { Hydrothermal vein } \\
\text { Hydrothermal vein }\end{array}$ & $\begin{array}{c}\mathrm{K} \\
\mathrm{Kv} \\
\mathbf{K}\end{array}$ & HS & $\begin{array}{l}200 \\
286 \\
145\end{array}$ & $\begin{array}{l}2.39 \\
2.66 \\
1.02\end{array}$ & $\begin{array}{l}0.03 \\
\text { n.d. } \\
0.04\end{array}$ \\
\hline Tugtupite & 64750 & Hydrothermal vein & $\mathrm{T}$ & & 3.9 & n.d. & 0.01 \\
\hline $\begin{array}{l}\text { Ussingite } \\
\text { Ussingite }\end{array}$ & $\begin{array}{l}65914 \\
163056\end{array}$ & $\begin{array}{l}\text { Hydrothermal vein } \\
\text { Ussingite vein in } \\
\text { recrystallized naujaite }\end{array}$ & $\begin{array}{l}\mathrm{Kv} \\
\mathrm{Tu}\end{array}$ & & $\begin{array}{l}3.6 \\
98\end{array}$ & $\begin{array}{l}0.07 \\
\text { n.d. }\end{array}$ & $\begin{array}{l}0.02 \\
0.03\end{array}$ \\
\hline Villiaumite & $119439-393.20$ & Arfvedsonite lujavrite & $\mathrm{Kv}$ & & 221 & - & n.d. \\
\hline
\end{tabular}

Sample numbers: five- and six-figure numbers are GGU numbers; other numbers and letters are sample numbers of individual collectors; *108107-191 means drill hole 7, $191 \mathrm{~m}$ depth (all 1081-drill holes are located outside Kvanefjeld); 119437-092.28 means drill hole $37,92.28 \mathrm{~m}$ depth (all 1194-drill holes are located on the Kvanefjeld).

+) Localities: K Kangerluarsuk area; Kv Kvanefjeld area; T Taseq area; Tu Tunulliarfik area.

${ }^{++}$Collectors: JE John Engell; OL Ole Larsen; LL Laust Løkkegaard; HM Harry Micheelsen; HS Henning Sørensen; other samples collected by the authors.

- not determined; F n.d. not detected ( $<0.05 \%)$; $\mathrm{Mg}$ n.d. not detected $(<0.01 \%)$.

Gerasimovskite, $\mathrm{TiNb}(\mathrm{OH})_{9}$, with $1.24 \% \mathrm{Li}$ is a weathering product of epistolite in ussingite veins of the Taseq area, north of Tunulliarfik.

Astrophyllite, $(\mathrm{K}, \mathrm{Na})_{3}(\mathrm{Fe}, \mathrm{Mn})_{7}(\mathrm{Ti}, \mathrm{Zr})_{2} \mid \mathrm{Si}_{8}$ $(\mathrm{O}, \mathrm{OH})_{31}$, with $0.30 \% \mathrm{Li}$ is found in considerable amounts in the border pegmatite of the kakortokites. The Ilimaussaq astrophyllite is an intermediate member of the $\mathrm{Ti}-\mathrm{Nb}$ substitution series between astrophyllite and niobophyllite (Semenov 1969).

The alkaline rocks of the Lovozero massif (Kola, Russia) contain other Li-rich micas - tainiolite and biotite (Semenov 1959; Vlasov et al. 1966). Equivalent minerals have not been confidently reported from the Ilímaussaq intrusion.

\section{Li crystallochemistry}

Lithium exhibits mixed crystallochemical properties (Cocco et al. 1969).

(1) $\mathrm{Li}-\mathrm{O}$ bonds. Because of its small ionic radius, $\mathrm{Li}^{+}$ $(0.82 \AA)$ tends to follow $\mathrm{Mg}^{2+}(0.80 \AA)$, and to a lesser extent $\mathrm{Fe}^{2+}(0.86 \AA)$ and $\mathrm{Al}^{3+}(0.61 \AA)$, into octahedral sites of silicate structures. However, $\mathrm{Li}^{+}$does not simply substitute for $\mathrm{Mg}^{2+}$ etc. but all these ions compete to enter ferromagnesian minerals where the optimal size of the relevant site varies slightly (Jensen.1973): clinopyroxene $(0.79 \AA)$, orthopyroxene and hornblende $(0.78 \AA)$, olivine $(0.77 \AA)$ and biotite $(0.76 \AA)$. Entry of variously charged ions into these optimal sites must satisfy charge balancing within the mineral and this has been confirmed by analysis. Thus plots of $2 \mathrm{Mg}^{2+}$ versus $\mathrm{Li}^{+}+\mathrm{Al}^{3+}$ in many Li-bearing pyroxenes, amphiboles and micas lie close to a 1:1 negative correlation line. $\mathrm{Li}^{\mathrm{VI}}+\mathrm{Si}^{\mathrm{IV}}=\left(\mathrm{Fe}^{2+}\right)^{\mathrm{VI}}+\mathrm{Al}^{\mathrm{IV}}$ and other substitution mechanisms have also been suggested (Henderson et al. 1989).

Coordination octahedra around $\mathrm{Li}^{+}$can occur either separately or linked together in various ways. The average $\mathrm{Li}^{(\mathrm{VI})}$-O bond length is $2.14 \AA$. $\mathrm{Li}$ in tetrahedral coordination with $\mathrm{O}$ is less important in nature but does occur in petalite. The Li-phosphate amblygonite has an intermediate coordination number.

(2) Li-F bonds. Li readily loses it outermost electron resulting in an extremely reactive chemical character. $\mathrm{Li}$-halide bonds are very stable. In the $\mathrm{NaCl}$ structure type, which includes the Ilímaussaq mineral villiaumite 
$(\mathrm{NaF}), \mathrm{Li}^{+}$is in 6-fold coordination and the $\mathrm{Li}-\mathrm{F}$ bond length is $2.014 \AA$. $\mathrm{LiF}_{4}$ tetrahedra, structurally equivalent to $\mathrm{SiF}_{4}$ tetrahedra, occur in phenakite and cryolithionite.

(3) $\mathrm{Li}-\mathrm{Na}$ and $\mathrm{Li}-\mathrm{K}$ substitution. Because of the small size of $\mathrm{Li}^{+}\left(0.82^{\mathrm{VI}} \AA\right)$ with respect to that of the next alkaline ions $\mathrm{Na}^{+}\left(1.10^{\mathrm{VI}} \AA, 1.24^{\mathrm{VII}} \AA\right)$ and $\mathrm{K}^{+}\left(1.46^{\mathrm{VI}}\right.$ $\AA, 1.59^{\mathrm{VIII}} \AA, 1.68^{\mathrm{XII}} \AA$ ), only limited substitution is expected. However, experimental work shows that in high-Al micas interlayer $\mathrm{Li}$ may replace about one-third of $\mathrm{K}^{+}$though natural examples have not yet been recorded (Robert et al. 1983).

Trace element geochemical studies have confirmed these crystallochemical controls and indicated their varying importance in different rock types. Thus $\mathrm{Li}-\mathrm{Na}$ substitution in plagioclase is probably responsible for more than $50 \%$ of the $\mathrm{Li}$ in dioritic rocks (Vorontsov and $\mathrm{Lin}$ 1966), whereas $\mathrm{Li}-\left(\mathrm{Fe}^{2+}, \mathrm{Mg}\right)$ substitution is dominant in the main $\mathrm{Li}$ carriers (hornblende, biotite, muscovite) of many granites. In this evolution from intermediate to acid rocks, the proportion of $\mathrm{Li}-\mathrm{O}$ bonds decreases relative to the abundance of $\mathrm{Li}-\mathrm{F}$ and $\mathrm{Li}-\mathrm{OH}$ bonds. Némec (1969) found that $\mathrm{F}$ values in pegmatitic muscovites generally correlate with $\mathrm{Li}$ values. Bailey (1977) has summarised the close geochemical correlation between $\mathrm{Li}$ and $\mathrm{F}$ in granitic rocks and minerals; both elements lower granite solidus temperatures and promote water solubility.

\section{Analytical methods}

$\mathrm{Li}$ and $\mathrm{F}$ were analysed by instrumental neutron activation using a fast transfer system and Cerenkov counting coupled to the Danish Nuclear Research Reactor DR2, Risø National Laboratory (Heydorn et al. 1977; Bailey et al. 1981b)). For $\mathrm{Li}$, empirical values for accuracy, precision and lower limit of detection were found to be $\pm 10-20 \%, \pm 10-15 \%$ and $0.2 \mathrm{ppm}$, respectively. The counting techniques were optimised for $\mathrm{Li}$, and $\mathrm{F}$ results can only be considered accurate to approximately $\pm 20 \%$. The detection limit for $\mathrm{F}$ is about $500 \mathrm{ppm}$. $\mathrm{Mg}$ was analysed either directly on powder pellets of the minerals using conventional X-ray fluorescence techniques or using atomic absorption analysis after HF dissolution. Values agree within $0.01 \%$ for the two techniques and the detection limit is $0.01 \% \mathrm{Mg}$.

$A$ few additional $\mathrm{Li}$ and $\mathrm{F}$ values were determined by atomic absorption analysis after HF dissolution, and a combined distillation-spectrophotometry technique, respectively. In the latter technique, powdered material was fused with sodium carbonate and subjected to steam distillation from sulphuric acid. Fluorine was extracted on anion resin, selectively eluted with a sodium acetate solution and measured on a Zeiss spectrophotometer (Hansen 1983).
Mineral separation from fine and medium grained rocks was effected by a combination of heavy liquid and magnetic techniques. Coarse material was often amenable to hand picking followed by further crushing and inspection under a binocular microscope. Percentages and types of impurities, up to the $2 \%$ level, were estimated and Li contents correspondingly adjusted.

\section{Results}

Results for $\mathrm{Li}, \mathrm{F}$ and $\mathrm{Mg}$ on 120 Ilímaussaq mineral samples ( 30 species) are presented in Table 2.

\section{Arfvedsonite}

Among the rock-forming minerals, arfvedsonite contains the highest amount of Li (225-2520 ppm). Arfvedsonite $\mathrm{Li}$ contents increase from $225-462$ ppm in sodalite foyaite and naujaite, to $378-725 \mathrm{ppm}$ in kakortokite, and reach a maximum of $1970-2520 \mathrm{ppm}$ in aegirine lujavrite I, the earliest of the lujavrite varieties. Arfvedsonites from later lujavrites tend to contain less $\mathrm{Li}$, even though whole-rock values are the highest found in the Ilimaussaq intrusion.

Semenov (1969) found that arfvedsonite from a raremetal naujaite pegmatite (with eudialyte, epistolite and chkalovite) contained $0.093 \% \mathrm{Li}$ and $0.80 \% \mathrm{~F}$. But in naujaite pegmatites free of rare-metal mineralization (i.e. with microcline, sodalite and arfvedsonite) the arfvedsonite only contained $0.009 \% \mathrm{Li}$ and $0.29 \% \mathrm{~F}$.

Arfvedsonite crystallizes late in sodalite foyaite and naujaite but is an early phase in kakortokites and arfvedsonite lujavrites. When arfvedsonites from the same crystallization stage are compared, then $\mathrm{Li}$ contents are always higher in arfvedsonites from the rock type crystallising later in the Ilímaussaq sequence. It thus seems likely that $\mathrm{Li}$ contents increase progressively in the late magmas. The smoothness of this increase could be interrupted if there were extensive fractionation of Li-rich arfvedsonite but there is no clear evidence for such an effect at present.

The maximal values in arfvedsonites from aegirine lujavrite I probably arise from the late crystallization of the arfvedsonite in this cumulate rock type. According to Bailey and Gwozdz (submitted), the average wholerock $\mathrm{Li}$ value for aegirine lujavrite $\mathrm{I}$ is only about 200 ppm due to the dilution by the Li-poor cumulus phases. The interstitial material is considered to represent the trapped coexisting magma and contains $540 \mathrm{ppm} \mathrm{Li}$. The interstitial arfvedsonite thus crystallised from a $\mathrm{Li}$ rich magma and has very high $\mathrm{Li}$ contents.

Arfvedsonite is probably the main Li carrier in most of the agpaitic rocks at Ilimaussaq, though this has only been checked quantitatively for aegirine lujavrite I. Bailey and Gwozdz (submitted) found that, for three sam- 


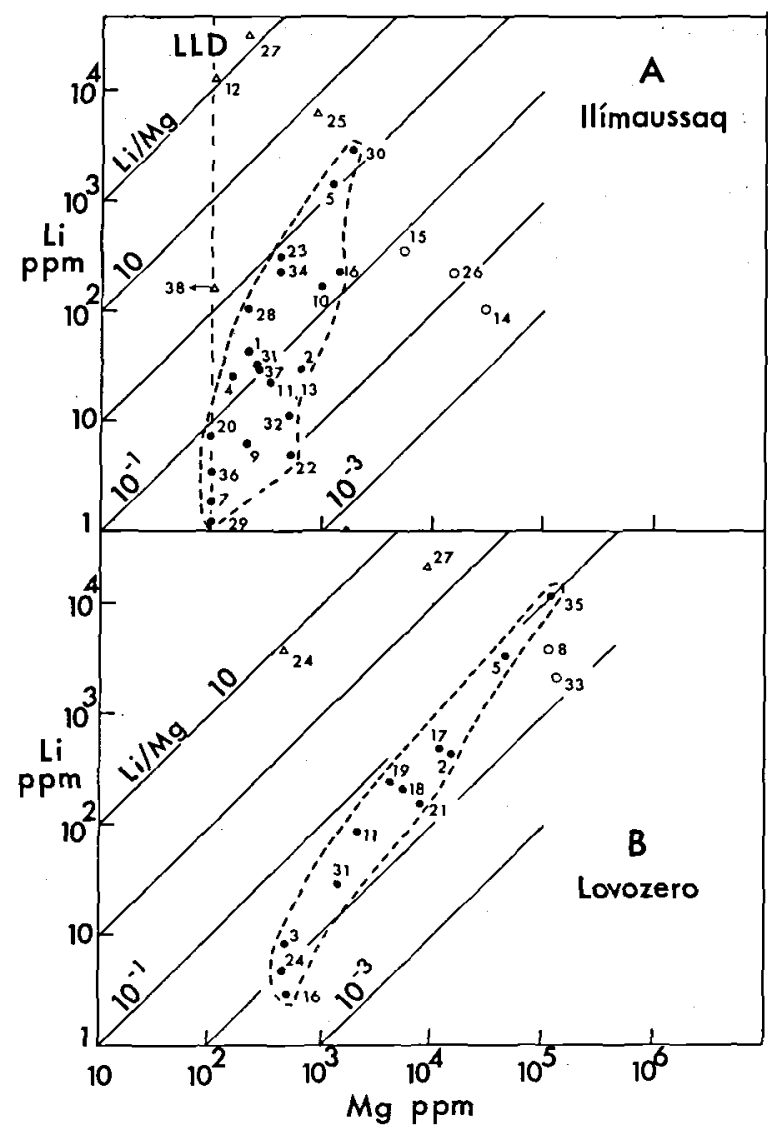

Fig. 2. $\mathrm{Li}-\mathrm{Mg}$ relations for minerals from the Ilimaussaq (A) and Lovozero (B) alkaline intrusions. Only median values are plotted. Closed circles - main group; triangles - high $\mathbf{L i} / \mathbf{M g}$ minerals; open circles - low $\mathrm{Li} / \mathrm{Mg}$ minerals. LLD lower limit of detection. Data for Ilímaussaq from Tables 1 and 2, this paper. Data for Lovozero from Semenov $(1959,1969)$, Gerasimovsky \& Kholina (1965), Gerasimovsky et al. (1966), Vlasov et al. (1966). Key to numbers: 1 acmite, 2 aegirine, 3 analcime, 4 analcime + natrolite, 5 arfvedsonite, 6 astrophyllite, 7 betanatrolite, 8 biotite, 9 chkalovite, 10 epistolite, 11 eudialyte, 12 gerasimovskite, 13 igdloite, 14 kaersutite, 15 kataphorite, 16 K-Na feldspar, 17 kupletskite, 18 labuntsovite, 19 lamprophyllite, 20 microcline + nepheline, 21 murmanite, 22 naujakasite, 23 nenadkevichite, 24 nepheline, 25 neptunite, 26 niobophyllite, 27 polylithionite, 28 pyrochlore, 29 quartz, 30 riebeckite, 31 sodalite, 32 sorensenite, 33 spodiophyllite, 34 steenstrupine, 35 tainiolite, 36 tugtupite, 37 ussingite, 38 villiaumite.

ples of aegirine lujavrite I, the arfvedsonite contained 67 to $91 \%$ of the total $\mathrm{Li}$.

There is a tendency for the $\mathrm{F}$ and $\mathrm{Mg}$ contents of the analysed arfvedsonites to decrease during the Ilímaussaq evolution (Table 2). Larsen (1976) also found a decrease for $\mathrm{Mg}$ among the Ilímaussaq amphiboles, though $\mathrm{Mg}$ values in early kakortokitic amphiboles are higher than in the preceding naujaites. Gerasimovsky et al. (1966: Table 125) found that $F$ contents of Lovozero amphiboles increased from phase I $(1.80 \%)$ to phase II (mainly $2.4 \%$ ) but fell slightly in phase III $(2.01 \%)$.

\section{Other minerals}

The highest contents for $\mathbf{L i}$ in Ilímaussaq minerals are found for polylithionite (2.97-3.54\%) and overlap previously published results. High values of $\mathrm{Li}$ have been confirmed in neptunite (5440-6700 ppm) and have been recorded for the first time in gerasimovskite $(1.24 \%)$.

Up to several hundred ppm Li are commonly found in the $\mathrm{Zr}, \mathrm{Nb}$, Ti and Th silicates: astrophyllite, epistolite, nenadkevichite, niobophyllite, pyrochlore and steenstrupine. Niobophyllite and astrophyllite probably form a solid-solution series at Ilímaussaq (Semenov 1969) and it is notable that astrophyllite, which is the endmember rich in alkalis, also has a high level of $\mathrm{Li}(3000$ ppm) compared to niobophyllite (157-218 ppm). However, a second astrophyllite sample only contains 223 $\mathrm{ppm} \mathrm{Li}$. This recalls the wide range recorded by Ganzeyev et al. (1969) who found $\mathrm{Li}$ values ranging from $130-5900 \mathrm{ppm}$ for astrophyllite.

Surprisingly, villiaumite, whose structure and chem-

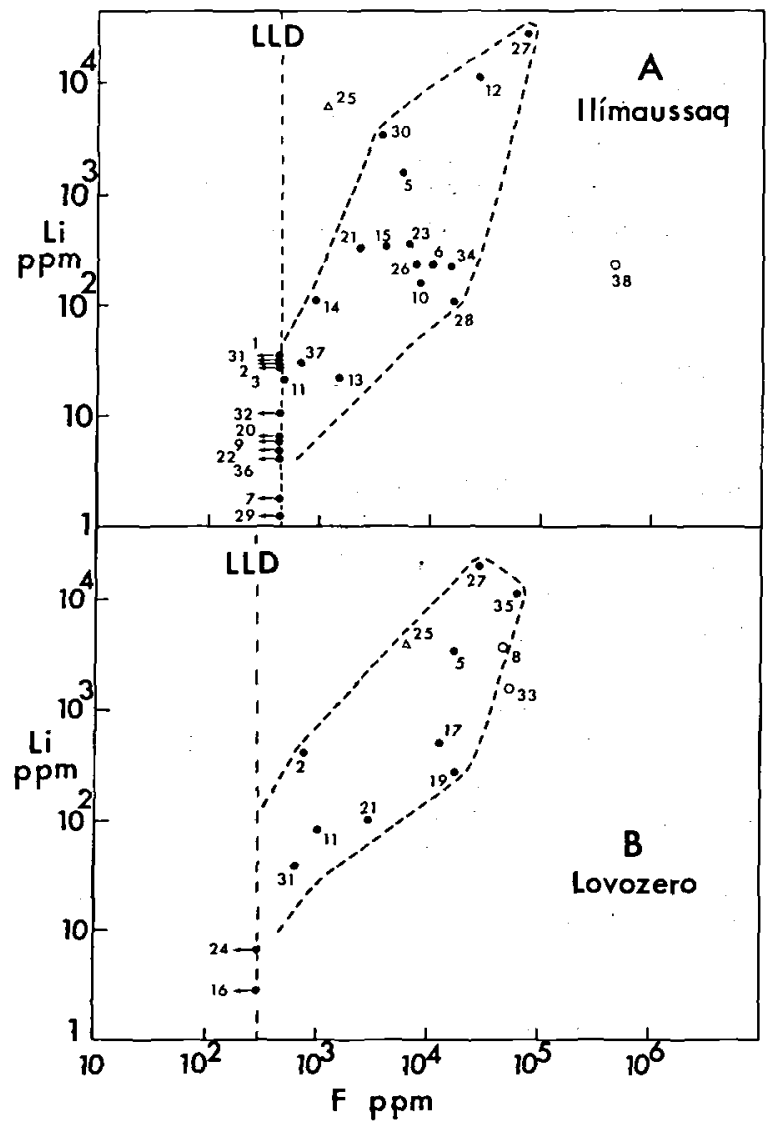

Fig. 3. Li-F relations for minerals from the Ilimaussaq (A) and Lovozero (B) alkaline intrusions. Only median values are plotted. Closed circles - main group; triangles - high Li/F minerals; open circles - low $\mathrm{Li} / \mathrm{F}$ minerals. Data sources and key to numbers as in Fig. 2. 
istry might be expected to readily incorporate $\mathrm{Li}$, contains only 221 ppm.

Except for arfvedsonite, there are no rock-forming minerals which consistently contain more than $50 \mathrm{ppm}$ Li. Aegirine (10-37 ppm), eudialyte (generally 7.5-32 ppm), microcline + nepheline $(2.0-8.2 \mathrm{ppm})$, sodalite (24-33 ppm) and zeolites (3.8-38 ppm) all have distinctly low Li contents when the Li-rich nature of the intrusion is considered. They are also poor in F.

$\mathrm{Li}$ values in aegirine are unrelated to stratigraphic height in a $200 \mathrm{~m}$ drill core through aegirine lujavrite I (Bailey and Gwozdz, submitted) but show a closer correlation to the percentage of interstitial, coexisting magma in each sample.

Acmite, commonly developed after aegirine or arfvedsonite at Ilímaussaq, shows similar Li contents to aegirine (Table 2).

Eudialyte exhibits high levels of $\mathrm{Li}(174-268 \mathrm{ppm})$ in the early sodalite foyaites, but levels fall to $12-17 \mathrm{ppm}$ in the succeeding naujaite and 7.1-18 ppm in kakortites. Similar low values are found in the deeper levels of the $200 \mathrm{~m}$ drill core through aegirine lujavrite I but the values rise to $10-32 \mathrm{ppm}$ in the top $50 \mathrm{~m}$. The reasons for these variations are unknown as yet. Eudialyte crystallises late in sodalite foyaite and naujaite, but early in kakortokites and aegirine lujavrite $I$.

Early crystallising microclines and nephelines in aegirine lujavrite I only contain $2.0-8.2 \mathrm{ppm} \mathrm{Li}$ whereas the interstitial zeolites - analcime and natrolite - contain 15-38 ppm. This is in agreement with the build-up of $\mathrm{Li}$ in the interstitial, residual magma of this rock type.

Bailey and Gwozdz (submitted) have pointed out that the low values of $\mathrm{Li}$ in the early crystallising phases of aegirine lujavrite I must imply low Li partition coefficients between these phases and the coexisting magma (about $540 \mathrm{ppm} \mathrm{Li}$ ). Li thus shows a much greater preference for the melt phase and this may be an innate feature of alkali-, volatile-rich agpaitic melts (cf. Steenfelt and Bohse 1975).

The lowest $\mathrm{Li}$ values in Ilímaussaq minerals are found for the leucocratic phases - mainly alkali-bearing silicates. These include analcime (3.8-12 ppm), beta-natrolite $(1.7 \mathrm{ppm})$, chkalovite $(8.2 \mathrm{ppm})$, naujakasite $(5$ ppm), quartz (1.2 ppm), tugtupite (3.9 ppm) and ussingite $(3.6 \mathrm{ppm})$. Reliable results demand very high purity separation of these minerals. In some cases, we consider the lowest analytical values from several samples of one mineral to be the most reliable.

In general, the results of the present study confirm the previously published analyses on $\mathrm{Li}$ in Ilimaussaq minerals. The main differences are in the extrapolation of arfvedsonite values to higher levels in the later lujavritic rocks and the recognition of a group of $\mathrm{Zr}-\mathrm{Nb}$ Ti-Th silicates with hundreds of ppm Li. In addition, there is a reduction of $\mathrm{Li}$ contents in several leucocratic phases compared to earlier publications. We suspect that the investigated separates have not always been pure.

\section{$\mathrm{Li}-\mathrm{Mg}$ and $\mathrm{Li}-\mathrm{F}$ relations}

Li-Mg and Li-F relations for Ilímaussaq minerals are shown in Figs. 2A and 3A. Comparable plots for Lovozero (Kola, Russia) minerals are given in Figs. 2B and $3 \mathrm{~B}$. Only average values for minerals have been plotted.

Most of the minerals from Ilímaussaq and Lovozero fall within a major grouping with positive correlations between $\mathrm{Li}$ and $\mathrm{Mg}$, and between $\mathrm{Li}$ and $\mathrm{F}$. $\mathrm{Li} / \mathrm{Mg}$ ratios and, to a lesser degree, $\mathrm{Li} / \mathrm{F}$ ratios increase as contents of $\mathrm{Li}, \mathrm{Mg}$ and $\mathrm{F}$ increase. Contents of these elements tend to increase in the following sequence: alkali-bearing leucosilicates - $\mathrm{Ti}-\mathrm{Zr}-\mathrm{Nb}$ silicates - pyroxenes amphiboles - micas. Although not investigated in detail, Li shows similar, though weaker correlations with $\mathrm{Fe}^{2+}$ and $\mathrm{OH}$ (structural $\mathrm{H}_{2} \mathrm{O}$ ). A Li-Na correlation, however, cannot be demonstrated.

Outside the main grouping, we distinguish three small sets of minerals: (a) minerals with very high $\mathrm{Li} / \mathrm{Mg}$ and $\mathrm{Li} / \mathrm{F}$ ratios (neptunite, polylithionite at Lovozero), (b) minerals with distinctly low ratios (biotite and spodiophyllite at Lovozero) and (c) villiaumite with very high $\mathrm{Li} / \mathrm{Mg}$ and very low $\mathrm{Li} / \mathrm{F}$.

For all these groupings, and for virtually every mineral species, the Lovozero samples have higher $\mathrm{Li}$, $\mathrm{Mg}$ and $\mathrm{F}$ contents but the Ilímaussaq samples have clearly higher $\mathrm{Li} / \mathrm{Mg}$ and slightly higher $\mathrm{Li} / \mathrm{F}$ ratios. This is consistent with the whole-rock geochemical comparisons made by Gerasimovsky (1969).

The $\mathrm{Li} / \mathrm{Mg}$ ratios of rocks and minerals at Ilímaussaq are remarkably high. For example, polylithionite has an average $\mathrm{Li} / \mathrm{Mg}$ ratio of roughly 3 at Lovozero but 150 at Ilímaussaq. Arfvedsonite averages 0.054 at Lovozero and about 0.53 at Ilímaussaq. The ratio rises to about 3.7 in arfvedsonite from aegirine lujavrite $I$ and to 7.9 in the final $\mathrm{M}-\mathrm{C}$ lujavrite. In these late-stage arfvedsonites, $\mathrm{Li}$ can be classified as a minor element whereas $\mathrm{Mg}$ occurs at trace element levels. The low contents of $\mathrm{Mg}$ in the Ilímaussaq intrusion, especially at the agpaitic stage, probably explain the absence of the Mg-rich Lovozero mica, tainiolite.

Gerasimovsky et al. (1966) concluded that, for Lovozero minerals, $\mathrm{Li}$ is characterised by an association with $\mathrm{Mg}, \mathrm{Fe}^{2+}, \mathrm{Na}$ and $\mathrm{F}$. Only the first two elements are reported as controlling the mineralogical distribution of Li at Ilímaussaq (Gerasimovsky 1969).

\section{Discussion}

The $\mathrm{Li}-\mathrm{Mg}$ and $\mathrm{Li}-\mathrm{F}$ relations for Ilímaussaq and Lovozero minerals, as well as the similar occurrences of particular mineral species and associations, are consistent with similar origins and evolutions for these two peralkaline $\mathrm{SiO}_{2}$-undersaturated intrusions. This close 
relationship has been emphasised several times (a.o. Ussing 1912; Gerasimovsky et al. 1966; Semenov 1969; Sørensen 1969, 1970).

The mineralogical relationship of $\mathrm{Li}$ with $\mathrm{Mg}$ and $\mathrm{F}$ arises from two different crystallochemical controls. The $\mathrm{Li}-\mathrm{Mg}$ correlation, and probably also the $\mathrm{Li}-\mathrm{Fe}^{2+}$ correlation, is expected on the classic rules of diadochy proposed by Goldschmidt (1954). Because of its similar ionic radius but lower valency, $\mathrm{Li}^{+}$is admitted into $\mathrm{Mg}^{2+}$-bearing minerals. Because $\mathrm{Li}$ is admitted, the $\mathrm{Li} / \mathrm{Mg}$ ratio of a mineral species should increase during fractionation; this is well exemplified by the Ilímaussaq arfvedsonites. The Li-Mg correlation through most of the mineral species also points to replacement of $\mathrm{Mg}$ by Li.

Partition coefficient theory (see above) would emphasise that comparing the $\mathrm{Li}$ and $\mathrm{Mg}$ ionic radii deviations from the ideal site sizes in clinopyroxene (0.03:0.01 $\AA$ ), hornblende $(0.04: 0.02 \AA)$ and biotite (0.06:0.04 $\AA$ ) indicates that clinopyroxene greatly favours $\mathrm{Mg}$ occupancy rather than $\mathrm{Li}$ whereas biotite only slightly favours $\mathrm{Mg}$ rather than $\mathrm{Li}$. Thus $\mathrm{Li} / \mathrm{Mg}$ ratios are expected to increase in the sequence: pyroxene amphibole - mica, and this tendency is probably valid even for the particular pyroxenes, amphiboles and mica species found in the Ilímaussaq and Lovozero intrusions.

Tauson (1965) also emphasised that $\mathrm{Li} / \mathrm{Mg}$ ratios can be linked to crystallographic controls. For example, in micas the medium-sized cations (such as $\mathrm{Mg}, \mathrm{Fe}^{2+}, \mathrm{Li}$ ) form a sheet-like grouping and are bound in octahedral coordination with four $\mathrm{O}$ and two $(\mathrm{OH}, \mathrm{F})$ anions. This bonding is much weaker in amphiboles. Since $\mathrm{Li}-$ $(\mathrm{OH}, \mathrm{F})$ bonding is more stable than $\mathrm{Mg}-(\mathrm{OH}, \mathrm{F})$ bonding, the replacement of $\mathrm{Mg}$ by $\mathrm{Li}$ should occur more readily in micas than in amphiboles and $\mathrm{Li} / \mathrm{Mg}$ ratios will be accordingly higher. This feature is found both at Ilímaussaq and Lovozero.

The positive $\mathrm{Li}-\mathrm{Mg}$ correlation in the major grouping of mineral species at Ilímaussaq and Lovozero is the opposite to that observed for individual mineral series, e.g. arfvedsonite, or for isomorphous series where major element diadochy of $\mathrm{Li}$ and $\mathrm{Mg}$ occurs. Thus, in the two Lovozero mica series, (phlogopite)-spodiophyllite tainiolite and tainiolite-polylithionite, values for $\mathrm{Li}$ and $\mathrm{Li} / \mathrm{Mg}$ increase as $\mathrm{Mg}$ decreases (Semenov 1959). Heterovalent substitution of the types $\mathrm{Li}^{+}+(\mathrm{Fe}, \mathrm{Al})^{3+}$ $\rightarrow 2 \mathrm{Mg}^{2+}$ or $\mathrm{Li}^{+}+\mathrm{Si}^{4+} \rightarrow \mathrm{Mg}^{2+}+\mathrm{Al}^{3+}$ yields the negative correlation between $\mathrm{Li}$ and $\mathrm{Mg}$ which is visible in Fig. 2 for the Li-, Mg-rich minerals.

The increasing $\mathrm{Li} / \mathrm{Mg}$ ratios with increasing $\mathrm{Mg}$ contents in the main grouping of minerals probably reflect the increased levels of $\mathrm{F}$ in the $\mathrm{Mg}$-rich members of the group. $\mathrm{Li}^{+}$has a greater affinity than $\mathrm{Mg}^{2+}$ for halogens, particularly $\mathrm{F}^{-}$, and should thus be preferentially incorporated in F-rich $\mathrm{Mg}^{2+}$-bearing minerals. Li-F linkages probably develop within the silicate melt and pass over into crystallising phases (Kogarko et al. 1968).
High $\mathrm{F}$ contents in magmas are known to promote the stability of Li-F micas at the expense of normal micas (Rieder 1971).

The coherent $\mathrm{Li}-\mathrm{Mg}$ relation through the main group of minerals suggests that the same controls will also apply to the Li-, Mg- and F-poor phases, the alkalibearing leucosilicates. There is no clear evidence that $\mathrm{Li}$ replaces $\mathrm{Na}$ in these minerals as suggested by Goldschmidt et al. (1934).

Outside the main group of minerals, there are phases with distinctly different $\mathrm{Li}-\mathrm{Mg}$ and $\mathrm{Li}-\mathrm{F}$ relations. At Ilímaussaq and Lovozero, neptunite has distinctly high $\mathrm{Li} / \mathrm{Mg}$ and $\mathrm{Li} / \mathrm{F}$ ratios. This is probably due to the very unusual structure of this mineral. $\mathrm{Li}$ is an essential component, rather than a substitutional component, and the coordination octahedra around $\mathrm{Li}$ occur separately rather than linked together as in amphiboles and micas (Cannillo et al. 1966).

Very low $\mathrm{Li} / \mathrm{Mg}$ ratios are anticipated in the mafic phases of the early augite syenite as the Mg contents of these minerals are the highest at Ilimaussaq (Larsen $1976,1981)$ but whole-rock $\mathrm{Li}$ values are the lowest (about 23 ppm). Biotite from Lovozero possesses low $\mathrm{Li} / \mathrm{Mg}$ and low Li/F ratios (point 8 on Figs. $2 \mathrm{~B}$ and $3 \mathrm{~B}$ ).

In the mineral villiaumite, $\mathrm{NaF}$, which has a $\mathrm{NaCl}$ structure, $\mathrm{Na}$ (and probably $\mathrm{Li}$ ) is in octahedral coordination but the bond length is smaller than $\mathrm{Li}^{(\mathrm{VI})}-\mathrm{O}$ bonds. The $\mathrm{Li} / \mathrm{Mg}$ ratio of villiaumite is exceptionally high but the $\mathrm{Li} / \mathrm{F}$ ratio is exceptionally low (point 38 on Figs. 2A and 3A). Presumably there is a clash between the affinity of $\mathrm{Li}$ for $\mathrm{F}$ (greater than that of $\mathrm{Na}$ ) and its limited possibility of replacing the much larger $\mathrm{Na}$ ion.

Li-Na substitution more clearly occurs in cookeite. This mineral usually contains $1.2-2.1 \% \quad \mathrm{Li}$ and $0.0-1.3 \% \mathrm{Na}$ but at Ilímaussaq there is $0.23 \% \mathrm{Li}$ and $3.34 \% \mathrm{Na}$ (Semenov 1969).

\section{Li-rich geochemical associations}

The Ilímaussaq and Lovozero intrusions constitute a distinct, though rare, type of Li-rich association. Its most characteristic feature is the low content of Si in the rocks and minerals of the association. This results in the widespread substitution of $\mathrm{Li}$ for $\mathrm{Fe}^{2+}$ and $\mathrm{Mg}$ in agpaitic minerals (arfvedsonite, aegirine, eudialyte etc.) at Ilímaussaq $\left(\mathrm{Fe}^{2+}>>\mathrm{Mg}\right.$ ) and Lovozero $\left(\mathrm{Fe}^{2+}>\mathrm{Mg}\right)$; there is only a weak development of independent $\mathrm{Li}$ minerals. A similar situation occurs in certain Li-rich granites and greisens where there is again sufficient $\mathrm{Fe}^{2+}$ and $\mathrm{Mg}$ and the mineralogy is dominated by a variety of Li-F-bearing micas (Henderson et al. 1989).

In major contrast to these two associations, there are certain Li-rich granite pegmatites and greisenised granites (Mulligan 1965; Anstett et al. 1990) which are characterised by very low $\mathrm{Fe}^{2+}$ and $\mathrm{Mg}$ contents and the development of many $\mathrm{Li}$ phases - spodumene, ambly- 
gonite, lepidolite, zinnwaldite, petalite, cryolithionite, triphylite-lithiophilite and others. Some of these occurrences are commercially valuable due to the large tonnages of $\mathrm{Li}$ minerals. The low $\mathrm{Fe}$ and $\mathrm{Mg}$ character of this geochemical association minimises the substitution of $\mathrm{Li}$ and leads to its concentration in independent $\mathrm{Li}$ minerals.

\section{Acknowledgements}

We thank Professor H. Sørensen, Professor H. Micheelsen, J. Engell, S. Andersen, O. Larsen and the late L. Løkkegaard for the donation of mineral specimens, and the Geological Survey of Greenland and the Ris $\varnothing \mathrm{Na}$ tional Laboratory for access to drill core materials from the Ilímaussaq intrusion. H. Sørensen kindly gave a critical appraisal of the manuscript. The support of the $\mathrm{Li}$ and $\mathrm{F}$ analytical programmes by $\mathrm{K}$. Heydorn of the Risø National Laboratory and by the Danish Natural Science Research Council (SNF) is gratefully acknowledged. Publication of this paper was authorized by the Geological Survey of Greenland.

\section{Dansk sammendrag}

Lithium i mineraler fra den alkaline Ilímaussaq intrusion i Sydgrønland.

Polylithionit, neptunit, riebeckit, Na-cookeit, ephesit, gerasimovskit, astrophyllit og arfvedsonit indeholder op til over 0.5 vægt $\% \mathrm{Li}_{2} \mathrm{O}$. Hovedparten af intrusionens $\mathrm{Li}$ er bundet $\mathrm{i}$ arfvedsonit, - der generelt indeholder 200-2500 ppm Li. Li-indholdet afhænger af mineralernes struktur, deres indhold af $\mathrm{Mg}$ og $\mathrm{F}$ og fraktioneringsstadiet. Li er sandsynligvis knyttet til $\mathrm{F} \mathrm{i}$ fluidfasen, en relation, der fortsætter under krystallisationen, hvor $\mathrm{Li}$ substituerer $\mathrm{Mg}$. $\mathrm{Li} / \mathrm{Mg}$ og $\mathrm{Li} / \mathrm{F}$ er $\mathrm{i}$ Ilímaussaqs mineraler højere end for tilsvarende mineraler fra Lovozero intrusion, Kola halvøen.

\section{References}

Anstett, T.F., Krauss, U.H., Ober, J. A. \& Schmidt, H. W. 1990. International strategic minerals inventory summary report - lithium. U.S. Geol. Surv. Circ. 930-1: 28 pp.

Bailey, J.C. 1977. Fluorine in granitic rocks and melts: A review. Chem. Geol. 19: 1-42.

Bailey, J.C. \& Gwozdz, R. (submitted for publication): Li distribution in aegirine lujavrite, Ilímaussaq alkaline intrusion. South Greenland: role of cumulus and post-cumulus processes. Lithos.

Bailey, J. C., Larsen, L. M. \& Sørensen, H. 1981a. Introduction to the Ilimaussaq intrusion with a summary of the reported investigations. Rapp. Grønlands geol. Unders. 103: 5-17.

Bailey, J. C., Gwozdz, R., Rose-Hansen, J., Andersen, L. S.
\& Sørensen, H. 1981b. Distribution of lithium and fluorine in lujavrites from the Ilímaussaq intrusion. Rapp. Grønlands geol. Unders. 103: 81-86.

Bohse, H., Brooks, C. K. \& Kunzendorf, K. 1971. Field observations on the kakortokites of the Ilímaussaq intrusion, South Greenland. Rapp. Grønlands geol. Unders. 38: 43 pp.

Bøggild, O. B. 1953. The mineralogy of Greenland. Meddr. Gronland 149, 3: 422 pp.

Cannillo, E., Mazzi, F. \& Rossi, G. 1966. The crystal structure of neptunite. Acta Cryst. 21: 200-207.

Cocco, G., Fanfani, L., Zanazzi, P. F., Heier, K. S. \& Billings, G. K. 1969. Lithium. In Wedepohl, K. H. (editor) Handbook of Geochemistry, vol. II, Chapter 3. Springer, Berlin.

Drits, V. A. \& Semenov, E. I. 1975. Structural-mineralogical characteristics of ephesite from south Greenland. Trudy Miner. Muzei im A. E. Fersmana 24: 175-180.

Engell, J. 1973. A closed system crystal-fractionation model for the agpaitic Ilímaussaq intrusion, South Greenland with special reference to the lujavrites. Bull. geol. Soc. Denmark 22: 334-362.

Ferguson, J. 1964. Geology of the Ilímaussaq alkaline intrusion, South Greenland. Description of map and structure. Meddr. Grønland 172: 4, 1-82.

Ganzeyev, A. A., Yefimov, A.F. \& Semenov, N. G. 1969. Isomorphism of the alkali metals in minerals of the astrophyllite group. Geochem. Intern. 6: 295-300.

Gerasimovsky, V.I. 1969. Geochemistry of the Ilímaussaq alkaline massif (South-West Greenland). Izd. Nauka, Moskva (In Russian).

Gerasimovsky, V. I. \& Kholina, Yu. B. 1965. Geochemistry of $\mathrm{Li}$ in the nepheline syenites. In Khitarov, N. I. (editor) Problems in geochemistry. Izd. Nauka, Moskva (In Russian).

Gerasimovsky, V.I., Volkov, V. P., Kogarko, L. N., Polyakov, A. I., Saprykina, T. V. \& Balashov, Yu. A. 1966. The geochemistry of the Lovozero alkaline massif. Izd. Nauka, Moskva (In Russian).

Goldschmidt, V.M. 1954. Geochemistry. Oxford Univ. Press, London.

Goldschmidt, V. M., Bauer, H. \& Witte, H. 1934. Zur Geochemie der Alkalimetalle II. Nachr. Ges. Wiss. Göttingen, IV, N.F. 1: 39-55.

Hamilton, E. I. 1964. The geochemistry of the northern part of the Ilímaussaq intrusion, SW Greenland. Meddr. Grønland 162 (10): 104 pp.

Hansen, N. B. 1983. Kemisk Analyse af Mineraler og Bjergarter. ISBN 87-7375-0018. Geologisk Centralinstitut, Copenhagen, Denmark. 312 pp.

Heier, K.S. \& Adams, J. A.S. 1964. The geochemistry of the alkali metals. Phys. Chem. Earth 5: 253-381.

Henderson, C. M. B., Martin, J.S. \& Mason, R. A. 1989. Compositional relations in Li-micas from S. W. England and France: an ion- and electron-microprobe study. Min. Mag. 53: 427-449.

Heydorn, L., Skanborg, P. Z., Gwozdz, R., Schmidt, J. O. \& Wacks, M.E. 1977. Determination of lithium by instrumental neutron activation analysis. J. Radioanal. Chem. 37: $155-168$.

Jensen, B. B. 1973. Patterns of trace element partitioning. Geochim. Cosmochim. Acta 37: 2227-2242.

Kogarko, L. N., Krigman, L. D. \& Sharudilo, N. S. 1968. Experimental investigations of the effect of alkalinity of silicate melts on the separation of fluorine into the gas phase. Geochem. Intern. 5: 782-790.

Larsen, L.M. 1976. Clinopyroxenes and coexisting mafic minerals from the alkaline Ilímaussaq intrusion, South Greenland. J. Petrol. 17: 258-290.

Larsen, L. M. 1981. Chemistry of feldspars in the Ilímaussaq augite syenite with additional data on some other minerals. Rapp. Grønlands geol. Unders. 103: 31-37.

Larsen, L. M. \& Sørensen, H. 1987. The Ilímaussaq intrusionProgressive crystallization and formation of layering in agpaitic magma. In Fitton, J. G. \& Upton, B. G. J. (editors) 
Alkaline Igneous Rocks. Geological Society of London Special Publications 30: 473-488.

Makovicky, E. \& Karup-Møller, S. 1981. Crystalline steenstrupine from Tunugdliarfik in the Ilímaussaq alkaline intrusion, South Greenland. N. Jb. Miner. Abh. 140: 300-330.

Mulligan, R. 1965. Geology of the Canadian lithium deposits. Geol. Survey. Can., Econ, Geol. Rep. 21: 131 pp.

Némec, D. 1969. Fluorine in pegmatitic muscovites. Geochem. Intern. 6: 58-68.

Povarennykh, A.S., Platonov, A.N. \& Belichenko, V.P. 1970. On the colour of ussingite from the Ilímaussaq (South Greenland) and Lovozero (Kola Peninsula) alkaline intrusions. Bull. geol. Soc. Denmark 20: 20-26.

Rieder, M. 1971. Stability and physical properties of synthetic lithium-iron micas. Am. Miner. 56: 256-280.

Robert, J.-L., Volfinger, M., Barrandon, J.-N. \& Basutçu, M. 1983. Lithium in the interlayer space of synthetic trioctahedral micas. Chem. Geol. 40: 337-351.

Schaller, W. T., Carron, M. K. \& Fleischer, M. 1967. Ephesite, $\mathrm{Na}\left(\mathrm{LiAl}_{2}\right)\left(\mathrm{Al}_{2} \mathrm{Si}_{2}\right) \mathrm{O}_{10}(\mathrm{OH})_{2}$, a trioctahedral member of the margarite group, and related brittle micas. Am. Miner. 52: $1689-1696$.

Semenov, E.I. 1959. Lithium-bearing and other micas and hydromicas in the alkaline pegmatites of the Kola Peninsula. Trans. Min. Mus. Acad. Sci. USSR 9: 107-137 (In Russian).

Semenov, E. I. 1969. Mineralogy of the Ilímaussaq Alkaline Massif. Izd. Nauka, Moskva (In Russian).

Steenfelt, A. \& Bohse, H. 1975. Variations in the content of uranium in eudialyte from the differentiated alkaline Ilímaussaq intrusion, South Greenland. Lithos 8: 39-45.
Sørensen, H. 1962. On the occurrence of steenstrupine in the Ilímaussaq massif, Southwest Greenland. Meddr. Grønland 167, 1: $251 \mathrm{pp}$

Sørensen, H. 1969. Rhythmic igneous layering in peralkaline intrusions. An essay review on Ilímaussaq (Greenland) and Lovozero (Kola, USSR). Lithos 2: 261-283.

Sørensen, H. 1970. Internal structures and geological setting of the agpaitic intrusions - Khibina and Lovozero of the Kola peninsula and Ilímaussaq, South Greenland. Can. Miner. 10: 299-334.

Sørensen, H., Rose-Hansen, J., Nielsen, B. L., Løvborg, L., Sørensen, E. \& Lundgaard, T. 1974. The uranium deposit at Kvanefjeld, the Ilímaussaq intrusion, South Greenland. Geology, reserves, benefication. Rapp. Grønlands geol. Unders. 60: 54 pp.

Tauson, L. V. 1965. Factors in the distribution of the trace elements during the crystallization of magmas. Phys. Chem. Earth 6: 215-250.

Ussing, N. V. 1912. Geology of the country around Julianehåb, Greenland. Meddr. Gronland 38: $376 \mathrm{pp}$.

Vlasov, K. A., Kuzmenko, M. V. \& Es'kova, E. M. 1966. The Lovozero Alkali Massif. Oliver \& Boyd, Edinburgh and London. $627 \mathrm{pp}$.

Vorontsov, A. Ye. \& Lin, N. G. 1966. Rubidium and lithium in the granitoids of the Bugulmin complex (Eastern Sayans). Geochem. Intern. 3: 1108-1116.

Wilson, G. C. \& Long, J. V.P. 1983. The distribution of lithium in some Cornish minerals: ion microprobe measurements. Min. Mag. 47: 191-199. 